\title{
BMJ Open Prognostic impact of albumin-bilirubin score on the prediction of in-hospital mortality in patients with heart failure: a retrospective cohort study
}

Su Han (D) , Chuanhe Wang, Fei Tong, Ying Li, Zhichao Li, Zhaoqing Sun, Zhijun Sun

To cite: Han S, Wang C, Tong F, et al. Prognostic impact of albumin-bilirubin score on the prediction of in-hospital mortality in patients with heart failure: a retrospective cohort study. BMJ Open 2022;12:e049325. doi:10.1136/ bmjopen-2021-049325

\section{- Prepublication history and} additional supplemental material for this paper are available online. To view these files, please visit the journal online (http://dx.doi.org/10.1136/ bmjopen-2021-049325).

Received 23 January 2021 Accepted 01 December 2021

Check for updates

(C) Author(s) (or their employer(s)) 2022. Re-use permitted under CC BY-NC. No commercial re-use. See rights and permissions. Published by BMJ.

Department of Cardiology, Shengjing Hospital of China Medical University, Shenyang, Liaoning, China

Correspondence to Dr Zhijun Sun;

sunzj_99@163.com

\section{ABSTRACT}

Objectives Liver dysfunction is prevalent in patients with heart failure (HF) and can lead to poor prognosis. The albumin-bilirubin (ALBI) score is considered as an effective and convenient scoring system for assessing liver function. We analysed the correlation between ALBI and in-hospital mortality in patients with HF.

Design A retrospective cohort study.

Setting and participants A total of 9749 patients with HF (from January 2013 to December 2018) was enrolled and retrospectively analysed.

Main outcome measures The main outcome is inhospital mortality.

Results ALBI score was calculated using the formula $\left(\log _{10}\right.$ bilirubin [umol/L] * 0.66$)+($ albumin $[\mathrm{g} / \mathrm{L}]$ * $-0.085)$, and analysed as a continuous variable as well as according to three categories. Following adjustment for multivariate analysis, patients which occurred in-hospital death was remarkably elevated in tertile 3 group (ALBI $\geq 2.27$ ) (OR 1.671, 95\% Cl 1.228 to $2.274, p=0.001$ ), relative to the other two groups (tertile 1: $\leq 2.59$; tertile 2: -2.59 to -2.27 ). Considering ALBI score as a continuous variable, the in-hospital mortality among patients with HF increased by $8.2 \%$ for every 0.1-point increase in ALBI score (OR 1.082; $95 \% \mathrm{Cl} 1.052$ to $1.114 ; \mathrm{p}<0.001)$. The ALBI score for predicting in-hospital mortality under C-statistic was $0.650(95 \% \mathrm{Cl} 0.641$ to $0.660, \mathrm{p}<0.001)$ and the cut-off value of ALBI score was -2.32 with a specificity of 0.630 and a sensitivity of 0.632 . Moreover, ALBI score can enhance the predictive potential of NT-pro-BNP (NT-pro-BNP +ALBI vs NT-pro-BNP: Cstatistic: $z=1.990, p=0.0467$; net reclassification improvement $=0.4012, p<0.001$; integrated discrimination improvement $=0.0082, p<0.001$ ). Conclusions In patients with HF, the ALBI score was an independent prognosticator of in-hospital mortality. The predictive significance of NT-proBNP + ALBI score was superior to NT-proBNP, and ALBI score can enhance the predictive potential of NT-proBNP.

\section{INTRODUCTION}

Patients with heart failure (HF) usually have a poor quality of life and dismal prognosis. ${ }^{12}$ It
Strengths and limitations of this study

- This study explored the association between albumin-bilirubin (ALBI) score and in-hospital mortality in 9749 patients with heart failure(HF).

- This study compared the prognostic potential of ALBI score with NT-pro-BNP.

- This study also confirmed whether ALBI score can enhance the prognostic potential of NT-pro-BNP in HF patients.

- In this study, our data on serum alkaline phosphatase and $\gamma$-glutamyltransferase were not obtained, which might associate with the prognosis.

- The ALBI score was only calculated by baseline blood testing at admission, not through dynamic monitoring process at discharge, which might associate with long-term clinical outcomes in patients with HF.

is increasingly becoming clear that $\mathrm{HF}$ is not a single-organ disease. ${ }^{3-5}$ Liver dysfunction is prevalent in HF patients and it is mainly caused by liver hypoperfusion and hepatic congestion. ${ }^{36-8}$ Several studies have confirmed that liver dysfunction in $\mathrm{HF}$ patients patients with HF can worsen prognosis due to impairments in liver reserve function, possibly influencing the energy supply to heart. ${ }^{3}{ }^{6-11}$ Previous studies mostly measured the single parameter, such as bilirubin, albumin, alanine aminotransferase, etc to predict the HF prognosis. ${ }^{6}{ }^{12-14}$ However, single parameter can only present liver single function like inflammation, metabolism or synthesis, which can not reflect the liver reserve function comprehensively. ${ }^{15}$ In addition to conventional measures, a new approach, the albuminbilirubin (ALBI) score, has been considered as an important strategy to examine liver reserve function. ${ }^{15}{ }^{16}$ Previous studies have confirmed that the ALBI score is related to 
adverse events in patients with HF following discharge, but there are no similar studies assessing the relationship between the ALBI score and in-hospital events. ${ }^{7}$

In our study, we inspected whether the ALBI score is a significant clinical factor to estimate in-hospital mortality in patients with HF. Moreover, we verified whether the ALBI score could enhance the prognostic significance of n-terminal brain natriuretic peptide(NT-pro-BNP) for patients with HF.

\section{METHODS}

\section{Study design and setting}

Our retrospective study population comprised 11556 consecutive patients aged $>18$ years with $\mathrm{HF}$ as the main diagnosis on admission from ShengJing Hospital of China Medical University located in the northeastern part of China (from January 2013 to December 2018). HF was defined based on the modified Framingham criteria. ${ }^{17}$ We used a uniform questionnaire to collect the clinical and the procedural data of all the subjects. We employed the formula $\left(\log _{10}\right.$ bilirubin [umol/L] $\left.* 0.66\right)+($ albumin $[\mathrm{g} / \mathrm{L}] *-0.085)$ to calculate the ALBI score according to the serum albumin and total bilirubin levels at baseline. ${ }^{16}$ We collected samples of the venous blood from all the subjects on admission and kept them in standard tubes. Serum albumin and total bilirubin were assayed using completely automated enhanced immunonephelometric assay on a Beckman AU 5800 analyzer (Beckman Coulter, USA). The standard ranges for baseline albumin and total bilirubin are $35-53 \mathrm{~g} / \mathrm{L}$ and $3.4-20.5 \mathrm{umol} / \mathrm{L}$, respectively. The primary endpoint was all-cause in-hospital mortality.

Exclusion criteria were (1) acute myocardial infarction (492 cases); (2) chronic alcoholism (113 cases); (3) chronic kidney failure with dialysis and/or diagnosed liver disease on admission (483 cases); (4) prior history of cardiac transplantation (5 cases); (5) no albumin, no total bilirubin, or no NT-pro-BNP data (714 cases). We finally enrolled $9749 \mathrm{HF}$ subjects into the study. The mean hospitalisation period was $9.8 \pm 5.7$ days. Figure 1 exhibits the flow chart of selecting the patients. We clustered the subjects into three study groups as per the tertile of ALBI score on hospital admission (tertile 1: $\leq 2.59(\mathrm{n}=3250)$; tertile $2:-2.59$ to $-2.27(\mathrm{n}=3250)$; tertile $3: \geq 2.27(n=3249))$. This study accedes to the Declaration of Helsinki.

\section{Patient and public involvement}

No patients or public were involved in this study.

\section{Statistical analysis}

Normally distributed quantitative variables were presented as mean $\pm \mathrm{SD}$ and compared using the Kruskal-Wallis H-test, whereas non-normally distributed quantitative variables were presented as median (IQR) and employed the Mann-Whitney U-test to compare them. Categorical variables are presented as counts and proportions $(\%)$ and compared with $\chi^{2}$ test. When the number of variables was lower than 5 , Fisher's exact test was used to detect the differences. we performed the logistic univariate assessments to examine the prognosticators of in-hospital mortality (online supplemental appendices S1), and then all the variables were entered into the multivariate logistic regression model to uncover the independent prognosticators of in-hospital mortality. ALBI score was tested in the form of continuous variable and categorical variable. The output results were presented by as ORs with corresponding 95\% CIs. The prognostic potential of ALBI, NT-pro-BNP and NT-pro-BNP +ALBI was inspected using the discrimination indices as below: (1) A receiver operating characteristic curve and the area under the curve in connection with the in-hospital mortality were determined by MedCalc statistical software (V.18.1.1), ${ }^{18}$ (2) We got individual risk of in-hospital mortality by entering each model into a logistic regression model.

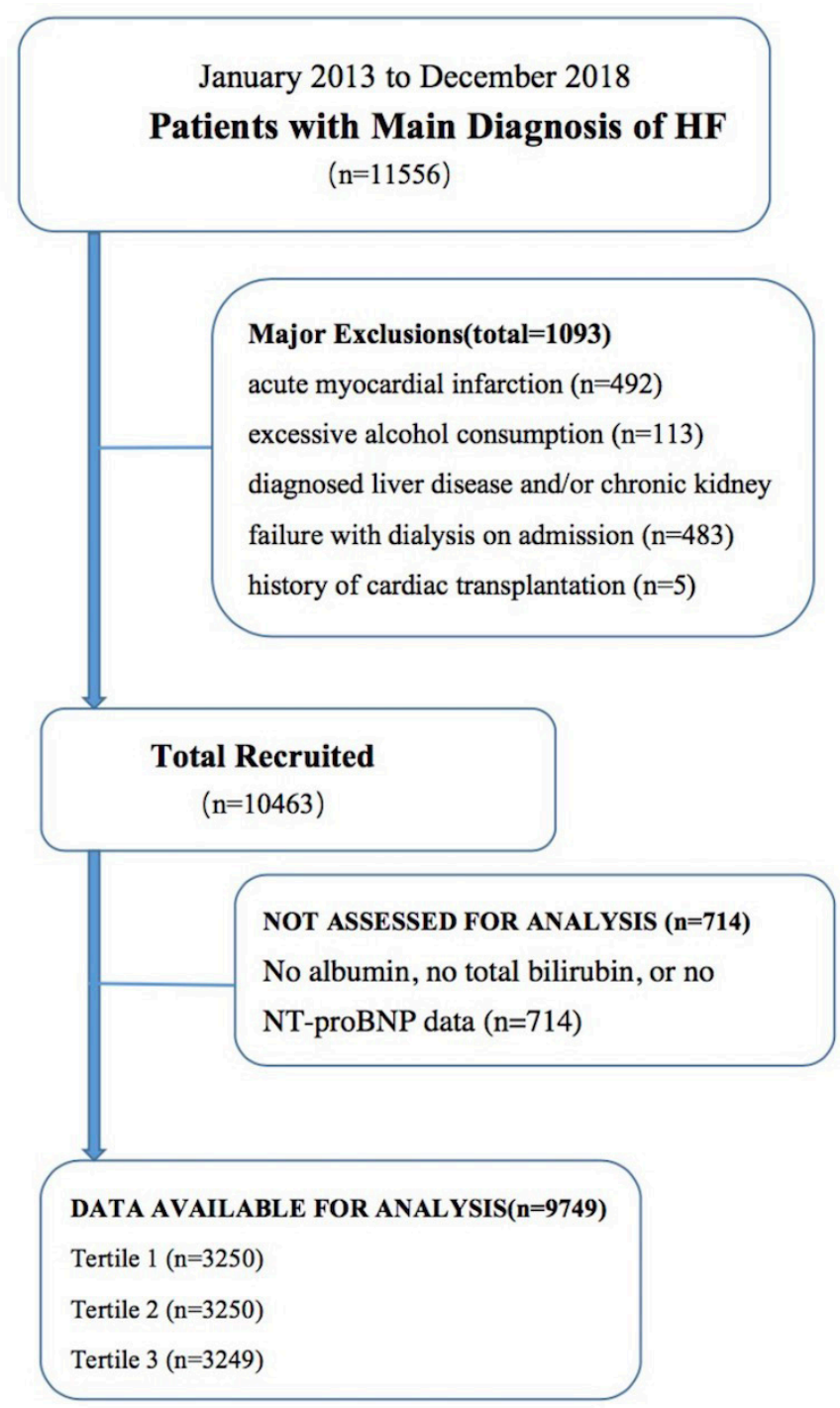

Figure 1 Flow chart of patient selection. HF, heart failure. NT-proBNP,n-terminal brain natriuretic peptide. 
ํํㄹำำำำ

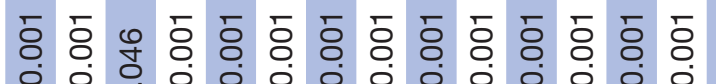

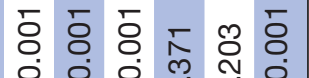
v i

V

崖

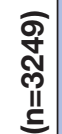

(1)

กิ่

ఝ

产

饮

궁

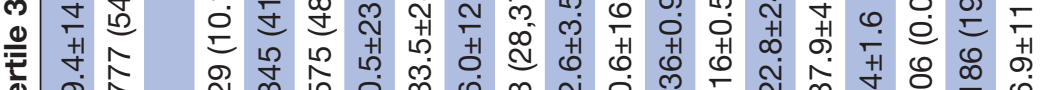

ᄋ

용 옹

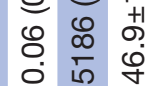

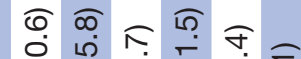

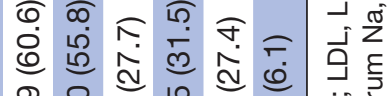

:

훙

흥ำ

을

ठ․

骨

-

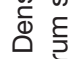

วิㅎํㅇ

离离

言 创

잉

సิ

.

\section{ले $\frac{\sigma}{\dot{\sigma}}$}

$\frac{\bar{m}}{\frac{1}{4}}$

$-\widehat{m}$

ลิธ

讨

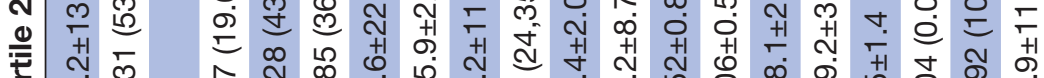

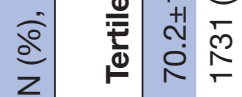

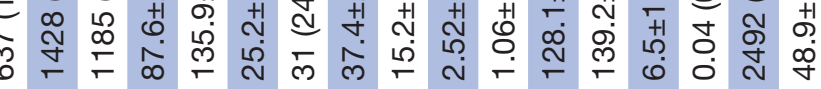

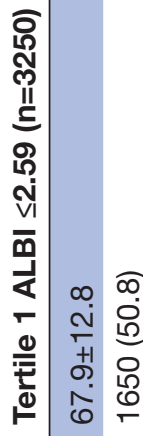

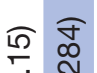

०. ले

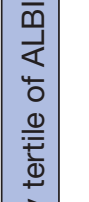

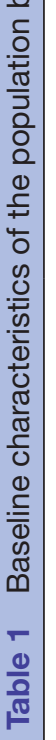

平度

ণุ

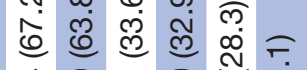

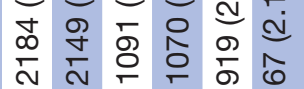

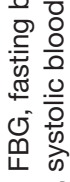

总望

ธุ ริ

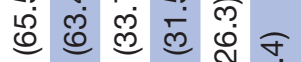

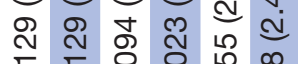

흐

这.

西

站

웅

$\stackrel{\vec{p}}{\vec{g}}$

हे

$\frac{\infty}{\overline{0}}$

$\mathscr{\infty}$

$\overrightarrow{0}$

$\overrightarrow{\vec{\omega}}$

흥.

$\stackrel{9}{9}$

กิ

定

O

竞

芯

ㅁ

言

등 홓

年

을 굴

这到

宽

is

응

هั

o

t?

敢 홍

둥

\section{จิ}

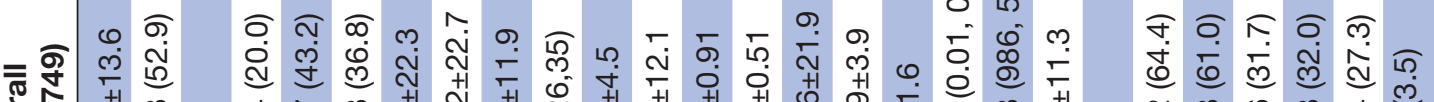

응 뚜워

웧ㄹ

O잉

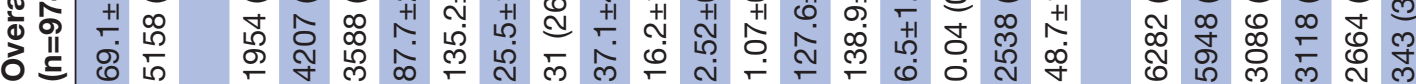

政

긍

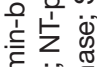
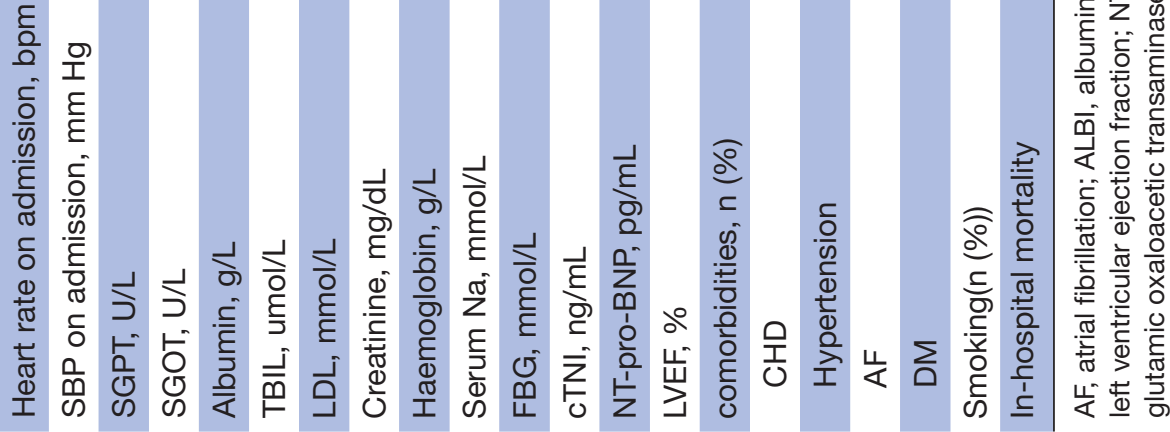

N

岕

足

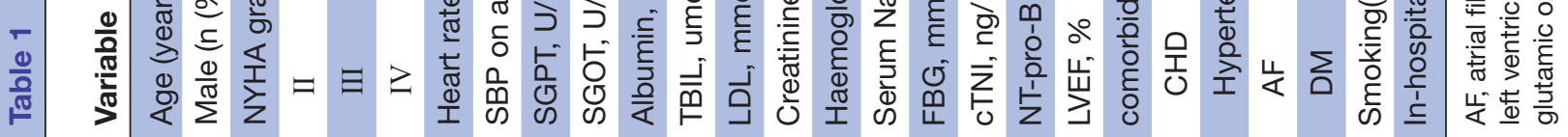


Table 2 Effects of multiple variables on clinical outcomes in univariate and multivariate analysis

\begin{tabular}{|c|c|c|c|c|c|c|}
\hline & \multicolumn{3}{|c|}{ Univariate analysis } & \multicolumn{3}{|c|}{ Multivariate analysis* } \\
\hline & OR & $95 \% \mathrm{Cl}$ & $P$ value & OR & $95 \% \mathrm{Cl}$ & $P$ value \\
\hline NT-pro-BNP per $100 \mathrm{pg} / \mathrm{mL}$ increase & 1.007 & 1.006 to 1.008 & $<0.001$ & & & \\
\hline ALBI, per 0.1 score increase & 1.135 & 1.108 to 1.163 & $<0.001$ & 1.082 & 1.052 to 1.114 & $<0.001$ \\
\hline \multicolumn{7}{|l|}{ ALBI as a categories variable } \\
\hline Tertile 2 & 1.167 & 0.839 to 1.624 & 0.358 & 0.863 & 0.612 to 1.212 & 0.398 \\
\hline Tertile 3 & 3.082 & 2.326 to 4.084 & $<0.001$ & 1.671 & 1.228 to 2.274 & 0.001 \\
\hline
\end{tabular}

*Adjusted for age, sex, NYHA grading, heart rate on admission, SBP on admission, SGPT, SGOT, LDL, creatinine, haemoglobin, serum Na, FBG, cTNI, LVEF, CHD, hypertension, AF, DM, smoking.

AF, atrial fibrillation; ALBI, albumin-bilirubin; CHD, coronary heart disease; cTNI, cardiac troponin I; DM, diabetes mellitus; FBG, fasting blood glucose; LDL, low-density lipoprotein; LVEF, left ventricular ejection fraction; NYHA, New York Heart Association; SBP, stylostic blood pressure; SGOT, serum glutamic oxaloacetic transaminase; SGPT, serum glutamate-pyruvate transaminase.

The Nagelkerke-R2, as well as the Hosmer-Lemeshow (HL) test from the regression model were employed as indices of goodness-of-fit of each risk model and to examine their calibration potential. ${ }^{19}$ We additionally computed the Brier scores of ALBI score, NT-pro-BNP and NT-pro-BNP +ALBI score. Lower Brier scores exhibited improved precision ${ }^{20}$ and (3) The absolute integrated discrimination improvement (IDI), as well as the category-free net reclassification improvement (NRI) were used to examine enhancements in risk estimation quantification of ALBI score and NT-pro-BNP +ALBI. ${ }^{21}$ All the statistical tests were two sided, and the statistical significance was marked by $\mathrm{p}<0.05$. We employed the Statistical Analysis Software (SAS Institute) V.9.4 to conduct all the statistical analysis.

\section{RESULTS}

\section{General characteristics}

The flow chart of patient selection was shown in figure 1. We finally enrolled a study cohort of $9749 \mathrm{HF}$ patients. The general characteristics were indicated in table 1 . The tertile 3 group had higher proportions of male patients and New York Heart Association (NYHA) grade IV than the other two groups. Moreover, patients in the tertile 3 group tended to have increasingly high heart rate, serum glutamatepyruvate transaminase (SGPT), serum glutamic oxaloacetic transaminase (SGOT), cTNI, total bilirubin, creatinine and NT-pro-BNP levels on admission. Systolic blood pressure, albumin, low density lipoprotein (LDL), fasting blood glucose(FBG), left ventricular ejection fraction (LVEF) in the tertile 3 group had a distinct diminishing pattern compared with the other groups. The proportion percentage of coronary heart disease (CHD), hypertension, atrial fibrillation (AF) and diabetes mellitus (DM) were markedly lower in the group of tertile 3 . Moreover, the tertile 3 group depicted the inclination of an elevated in-hospital mortality $(6.1 \%$ vs $2.1 \%$ and $2.4 \%, \mathrm{p}<0.001)$ (table 1$)$.

\section{Ability of ALBI score in prognosis estimation}

Numerous variables significantly influenced in-hospital mortality as observed on the univariate analysis supplemented online supplemental appendix S1: These variables were as follows: ALBI score, age, NYHA grading, heart rate on admission, systolic blood pressure on admission, SGPT, SGOT, creatinine, haemoglobin, Serum Na, FBG, cTNI, NT-proBNP, LVEF and the history of CHD, hypertension, AF, DM (online supplemental appendices S1).

The univariate analysis indicated that the ALBI score was related to the in-hospital mortality (OR 1.135, 95\% CI 1.108 to $1.163, \mathrm{p}<0.001$, for each 0.1-point increase) (table 2). Following covariate adjustments, the association remained present: the risk of in-hospital mortality increased by $8.2 \%$ for each 0.1-point increase in ALBI score (OR 1.082, 95\% CI 1.052 to $1.114, \mathrm{p}<0.001$ ) (table 2).

Even after the patients were divided into three groups, the ALBI score still significantly predicated incidence of in-hospital mortality (table 2). Under the univariate analysis, the tertile 3 group exhibited a markedly elevated risk of in-hospital mortality contrasted with the Tertile 1 and 2 groups (OR 3.082, 95\% CI 2.326 to 4.084, $\mathrm{p}<0.001$ ) (table 2). After adjustment for covariates, the group with the highest incidence of in-hospital mortality was still the tertile 3 (OR 1.671, 95\% CI 1.228 to $2.274, \mathrm{p}=0.001$ ) (table 2).

The predictive significance of ALBI, NT-pro-BNP and NT-pro-BNP +ALBI was assessed by C-statistic, which result were 0.650 (95\% CI 0.641 to 0.660 ), $0.652(95 \%$ CI 0.642 to 0.661 ) and 0.681 (95\% CI 0.672 to 0.690 ) (figure 2 and table 3), separately. The cut-off value for ALBI score was -2.32 with a sensitivity of 0.632 and a specificity of 0.630 . 


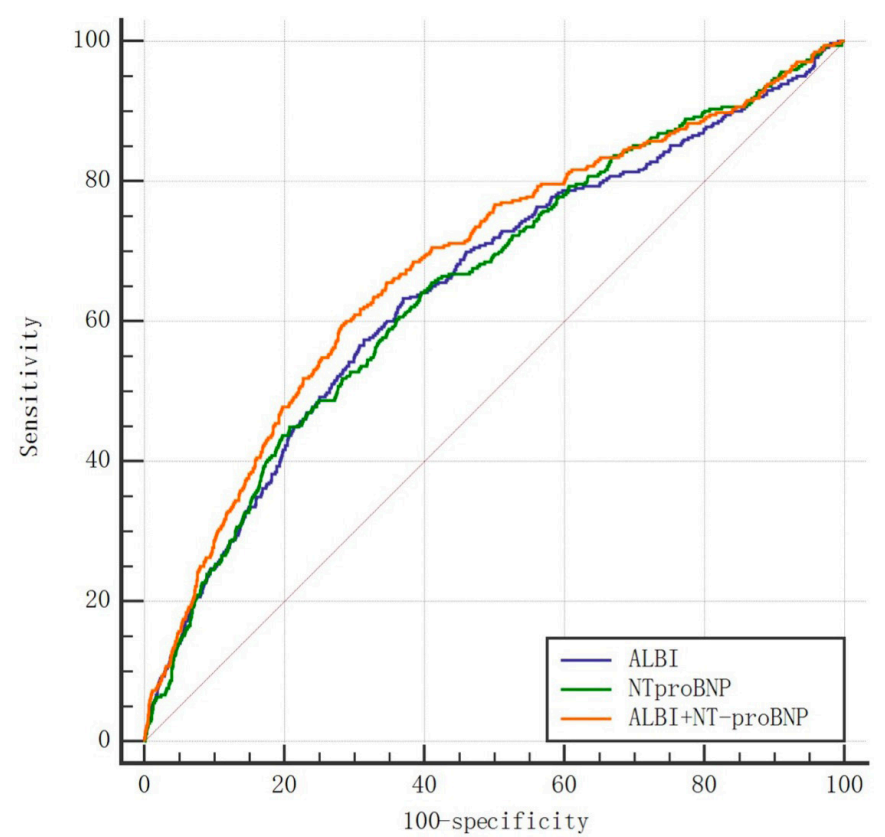

Figure 2 Receiver operating characteristic curves of ALBI, NT-pro-BNP and ALBI +NT-pro-BNP for in-hospital death prediction. ALBI, albumin-bilirubin.NT-proBNP,n-terminal brain natriuretic peptide.

\section{Improvement in prognostic significance of ALBI +NT-proBNP}

The HL p value, Nagelkerke-R2, as well as Brier score the of ALBI +NT-pro-BNP were Significantly better than the other two groups (table 3). The novel model in which the NT-pro-BNP was incorporated with ALBI can enhance the estimation significance. The prognostic value of NT-pro-BNP +ALBI was superior to that of NT-pro-BNP (C-statistic: $\mathrm{z}=1.990, \quad \mathrm{p}=0.0467 ; \quad \mathrm{IDI}=0.0082, \quad \mathrm{p}<0.001$; $\mathrm{NRI}=0.4012, \mathrm{p}<0.001)($ table 4$)$.

\section{DISCUSSION}

This study analysed the correlation between ALBI score and in-hospital mortality in patients with HF. We found that: (1) the ALBI score is an independent prognosticator of in-hospital death; (2) the predictive significance of NT-pro-BNP +ALBI score is superior to NT-pro-BNP, and ALBI score can enhance the estimation potential of the initial NT-pro-BNP model in patients with HF.

Various studies have assessed the prognostic clinical significance of distinct liver function test (LFT) indices in patients with HF. Post hoc evaluation of the Effificacy of Vasopressin Antagonism in Heart Failure Outcome Study with Tolvaptan(EVEREST) study posited that the low baseline albumin and increased bilirubin, were associated with clinical outcome. ${ }^{14}$ Moreover, the PROTECT study demonstrated that increased aspartate aminotransferase and alanine transaminase levels on day 3 of admission and decreased albumin levels on day 4 of admission are independent predictors of 180-day outcomes in patients with $\mathrm{HF}^{13}$ More and more studies have realised that the reserve of liver function is not only a single parameter, but also other factors with joint variables exist, so at present, the joint scoring system is mostly used to judge the liver function reserve of patients, including Child-Pugh classification (CP), MELD score and ALBI score. ${ }^{162223}$ CP has some disadvantages in that some of its parameters are subjective (ascites and encephalopathy), and inter-related indices (serum albumin and ascites), and it was not statistically established. ${ }^{24}$ MELD score system is an independent prediction index of adverse outcomes in patients with HF. ${ }^{25-29}$ Nevertheless, few studies on the ALBI score have been conducted. To the best of our knowledge, no study has explored the prediction value of the ALBI score for the in-hospital mortality in patients with HF. In our study, we elucidated that the ALBI score was correlated with in-hospital mortality for patients with HF. Considering the ALBI score as a continuous variable, we established that the risk of in-hospital mortality increased by $8.2 \%$ for each 0.1-point increase in ALBI (OR 1.082, 95\% CI 1.052 to $1.114, \mathrm{p}<0.001)$. As illustrated in table 2, ALBI score was still associated with in-hospital mortality when considered as a categorical variable (OR 1.671, 95\% CI 1.228 to $2.274, \mathrm{p}=0.001$ ). Previous reports have verified that NT-pro-BNP is linked to adverse events in HF patients, whether in hospital or discharged. ${ }^{3031}$ NT-pro-BNP is excreted by the kidney, and its circulating concentrations must be interpreted based on renal clearance. ${ }^{32}$ The patients with HF usually suffer from a renal dysfunction, ${ }^{3-5}$ which abnormally increases the concentrations of NT-pro-BNP, limiting its clinical utility. ${ }^{32}$ The ALBI score has no such restrictions, and the predictive value of ALBI score is not less than that NT-pro-BNP (C-statistic: $\mathrm{z}=0.0938$, $\mathrm{p}=0.9253)$. Furthermore, the ALBI score can enhance the predictive significance of NT-pro-BNP (C-statistic: $\mathrm{z}=1.990, \mathrm{p}=0.0467 ; \mathrm{IDI}=0.0082, \mathrm{p}<0.001 ; \mathrm{NRI}=0.4012$, $\mathrm{p}<0.001)$.

Table 3 NT-pro-BNP, NT-pro-BNP +ALBI and ALBI performance for the prognosis prediction

\begin{tabular}{|c|c|c|c|c|c|c|c|}
\hline & \multicolumn{4}{|c|}{ Discrimination } & \multicolumn{2}{|l|}{ Calibration } & \multirow{2}{*}{$\begin{array}{l}\text { Precision } \\
\text { Brier score }\end{array}$} \\
\hline & C-statistic & SE & $P$ value & $95 \% \mathrm{Cl}$ & HL P value & $\mathbf{R}^{2}$ & \\
\hline NT-pro-BNP & 0.652 & 0.0157 & $<0.001$ & 0.642 to 0.661 & 0.007 & 0.033 & 0.0336 \\
\hline ALBI & 0.650 & 0.0162 & $<0.001$ & 0.641 to 0.660 & 0.012 & 0.038 & 0.0335 \\
\hline
\end{tabular}

ALBI, albumin-bilirubin; HL, Hosmer-Lemeshow; NT-proBNP, n-terminal brain natriuretic peptide. 
Table 4 Comparisons of the predictive performance of NT-pro-BNP, NT-pro-BNP +ALBI and ALBI for the prognosis prediction

\begin{tabular}{llllccc}
\hline & Z for C-statistic & P for C-statistic & NRI & P for NRI & IDI & P for IDI \\
\hline ALBI vs NT-pro-BNP & 0.0938 & 0.9253 & 0.0518 & 0.3461 & 0.0018 & 0.4204 \\
ALBI+NT-pro-BNP vs NT-pro-BNP & 1.990 & 0.0467 & 0.4012 & $<0.001$ & 0.0082 & $<0.001$ \\
ALBI+NT-pro-BNP vs ALBI & 4.362 & $<0.001$ & 0.4054 & $<0.001$ & 0.0063 & 0.0001 \\
\hline
\end{tabular}

ALBI, albumin-bilirubin; IDI, integrated discrimination improvement; NRI, net reclassification improvement; NT-proBNP, n-terminal brain natriuretic peptide.

Although the detailed pathophysiological association between liver dysfunction and HF requires further assessments, there may be numerous mechanisms underlying this association. Severe congestive HF is related to two different kinds of liver conditions: acute hepatocellular necrosis that is caused by compromised blood supply as well as jaundice, which is correlated with the passive congestion. ${ }^{34}$ Compromised blood supply due to diminished cardiac output has a connection with acute hepatocellular necrosis with distinct escalations in serum aminotransferases. ${ }^{8-10}$ The passive hepatic congestion is associated with the elevated central venous pressure, resulting in increments in the levels of liver enzymes, as well as indirect and direct circulating bilirubin. Kato et al have studied the liver metabolism of HF in a rat model and established that congestive HF is linked to atypical metabolism in tissues adjacent to the heart. ${ }^{11}$ In the congestive HF rats, hepatic protein blood concentrations, including albumin, transferrin, retinol-binding protein and transthyretin were reduced and correlated with elevated levels of circulatory proinflammatory cytokines (tumor necrosis factor- $\alpha$ and interleukin-1 $\beta$ ). Because the heart has poor capacity of energy storage, and it needs a continuous energy supply, all the above studies support the possibility that liver dysfunction may lead to impaired cardiac energy supply, which may lead to a poor prognosis. ${ }^{1135}$

Although cardiogenic liver dysfunction was conventionally considered the result of cardiogenic shock, there is evidence that this is not the unique responsible incident. ${ }^{86}$ Our study comprised patients with acute exacerbation of chronic HF, and these patients may have a process of chronic congestion. Under such conditions, hepatocytes compensated impaired blood flow by increasing oxygen extraction. However, when patients occurred acute exacerbation of chronic HF with inadequate liver perfusion, this compensatory mechanism is exhausted, leading to hepatocellular hypoxia and necrosis. ${ }^{37}$ Thus, the patients having chronic congestion or portal hypertension, may exhibit acute cardiogenic liver injury even after mild circulatory disturbances. ${ }^{36}$ As shown in table 1 in our study, inadequate liver perfusion is manifested in two main aspects, one is lower SBP, and the other is a significantly reduced LVEF. Several studies have illustrated the complex relationship between lipid metabolism and liver dysfunction, and most of these studies described a decrease of total cholesterol, high density lipoprotein(HDL) and LDL cholesterol and triglycerides in patients with advanced liver disease. ${ }^{38-40}$ Furthermore, cholesterol homoeostasis was markedly disturbed in liver cirrhosis and total systemic cholesterol was negatively correlated with the Model of End-Stage Liver Disease(MELD) score. ${ }^{40}$ Our study showed similar results in table 1. This was mainly because genes involved in cholesterol biosynthesis declined in liver dysfunction, and were more strongly repressed in cirrhosis and hepatocellular carcinoma (HCC). ${ }^{39}$

The ALBI score was initially created from Japanese HCC patients to estimate the extent of liver dysfunction. ${ }^{16}$ However, it has also been widely used in patients without HCC. ${ }^{3541-43}$ It is worthy to note that one study has demonstrated that the ALBI score is related to liver function using the indocyanine green injection test. ${ }^{44}$ These results support that the ALBI score can reflect residual liver function reserve, even in patients without HCC.

Our findings have some clinical significance. First, observing ALBI in patients with HF may be significant in establishing HF patients with elevated risk of in-hospital adverse events. Moreover, the predictive value of the ALBI score was revealed to be the same as that of NT-pro-BNP. If the patient with HF has kidney dysfunction, where NT-pro-BNP has limited clinical utility, ALBI score becomes useful. Finally, consideration of both the cardiac function and liver dysfunction of patients may help clinicians more accurately determine in-hospital adverse event risk.

The current study has several limitations. First, it constituted a retrospective and observational design; therefore, possible confounders and selection bias were not absolutely adjusted. Accordingly, a larger scale multicentre validation study is warranted to confirm the relationship between ALBI score and in-hospital mortality in patients with HF. Second, despite excluding diagnosed liver diseases, liver dysfunction might have been happened by preexisting liver fibrosis due to non-HF aetiologies in some cases and we did not examine all the LFTs individually, as some biosignatures were missing in our dataset. For example, in the Finland risk (FINRISK) study, moderate to high levels of serum $\gamma$-glutamyltransferase were significantly correlated with $\mathrm{HF}$ 
incidence in a cohort of 38076 people. ${ }^{45}$ In addition, higher alkaline phosphatase was linked to a dismal prognosis in patients with AHF. ${ }^{46}$ Finally, ALBI score was only calculated by baseline blood testing at admission, lacking of dynamic monitoring process. Accordingly, further studies on the relationship between changes in ALBI score at discharge and long-term clinical outcomes in patients with HF are warranted.

\section{CONCLUSION}

In patients with HF, ALBI score was an independent prognosticator of in-hospital death. The predictive significance of NT-pro-BNP +ALBI was superior to NT-pro-BNP, and ALBI score can enhance the predictive potential of NT-pro-BNP.

Contributors SH designed of the work and and was a major contributor in writing the manuscript. CW, FT and YL collected and applied of statistical techniques to analyse study data. ZL and ZhaS managed activities to annotate (produce metadata), scrub data and maintain research data for initial use and later reuse. ZhiS formulated of overarching research goals and aims, and was responsible for the overall content. All authors read and approved the final manuscript.

Funding The authors have not declared a specific grant for this research from any funding agency in the public, commercial or not-for-profit sectors.

Competing interests None declared.

Patient consent for publication Not applicable.

Ethics approval This study involves human participants and was approved by Research Ethics Committee in the Shengjing Hospital of China Medical University no.2019PS594K. Participants gave informed consent to participate in the study before taking part.

Provenance and peer review Not commissioned; externally peer reviewed.

Data availability statement Data are available on reasonable request. Not applicable.

Supplemental material This content has been supplied by the author(s). It has not been vetted by BMJ Publishing Group Limited (BMJ) and may not have been peer-reviewed. Any opinions or recommendations discussed are solely those of the author(s) and are not endorsed by BMJ. BMJ disclaims all liability and responsibility arising from any reliance placed on the content. Where the content includes any translated material, BMJ does not warrant the accuracy and reliability of the translations (including but not limited to local regulations, clinical guidelines, terminology, drug names and drug dosages), and is not responsible for any error and/or omissions arising from translation and adaptation or otherwise.

Open access This is an open access article distributed in accordance with the Creative Commons Attribution Non Commercial (CC BY-NC 4.0) license, which permits others to distribute, remix, adapt, build upon this work non-commercially, and license their derivative works on different terms, provided the original work is properly cited, appropriate credit is given, any changes made indicated, and the use is non-commercial. See: http://creativecommons.org/licenses/by-nc/4.0/.

ORCID iD

Su Han http://orcid.org/0000-0002-2801-6982

\section{REFERENCES}

1 Chandra A, Vaduganathan M, Lewis EF, et al. Health-related quality of life in heart failure with preserved ejection fraction: the ParagonHF Trial. JACC Heart Fail 2019;7:862-74.

2 Ma L-Y, Chen W-W, Gao R-L, et al. China cardiovascular diseases report 2018: an updated summary. J Geriatr Cardiol 2020;17:1-8.

3 Harjola V-P, Mullens W, Banaszewski M, et al. Organ dysfunction, injury and failure in acute heart failure: from pathophysiology to diagnosis and management. A review on behalf of the acute heart failure Committee of the heart failure association (HFA) of the European Society of cardiology (ESC). Eur J Heart Fail 2017:19:821-36.

4 Peng J, Xiao X, Hu M, et al. Interaction between gut microbiome and cardiovascular disease. Life Sci 2018:214:153-7.

5 Rangaswami J, Bhalla V, Blair JEA, et al. Cardiorenal syndrome: classification, pathophysiology, diagnosis, and treatment strategies: a scientific statement from the American heart association. Circulation 2019;139:e840-78.

6 Suzuki K, Claggett B, Minamisawa M, et al. Liver function and prognosis, and influence of sacubitril/valsartan in patients with heart failure with reduced ejection fraction. Eur $J$ Heart Fail 2020;22:1662-71.

7 Matsue Y, Kagiyama N, Yamaguchi T, et al. Clinical and prognostic values of ALBI score in patients with acute heart failure. Heart Lung Circ 2020;29:1328-37.

8 Samsky MD, Patel CB, DeWald TA, et al. Cardiohepatic interactions in heart failure: an overview and clinical implications. J Am Coll Cardiol 2013;61:2397-405.

9 Laribi S, Mebazaa A. Cardiohepatic syndrome: liver injury in decompensated heart failure. Curr Heart Fail Rep 2014;11:236-40.

10 Alvarez AM, Mukherjee D. Liver abnormalities in cardiac diseases and heart failure. Int $J$ Angiol 2011;20:135-42.

11 Kato T, Niizuma S, Inuzuka Y, et al. Analysis of liver metabolism in a rat model of heart failure. Int $J$ Cardiol 2012;161:130-6.

12 Vasconcelos LABA, de Almeida EA, Bachur LF. Clinical evaluation and hepatic laboratory assessment in individuals with congestive heart failure. Arq Bras Cardiol 2007;88:590-5.

13 Biegus J, Hillege HL, Postmus D, et al. Abnormal liver function tests in acute heart failure: relationship with clinical characteristics and outcome in the protect study. Eur J Heart Fail 2016;18:830-9.

14 Ambrosy AP, Vaduganathan M, Huffman MD, et al. Clinical course and predictive value of liver function tests in patients hospitalized for worsening heart failure with reduced ejection fraction: an analysis of the Everest trial. Eur J Heart Fail 2012;14:302-11.

15 Pinato DJ, Sharma R, Allara E, et al. The ALBI grade provides objective hepatic reserve estimation across each BCLC stage of hepatocellular carcinoma. J Hepatol 2017;66:338-46.

16 Johnson PJ, Berhane S, Kagebayashi C, et al. Assessment of liver function in patients with hepatocellular carcinoma: a new evidencebased approach-the ALBI grade. J Clin Oncol 2015;33:550-8.

17 McKee PA, Castelli WP, McNamara PM, et al. The natural history of congestive heart failure: the Framingham study. N Engl J Med 1971;285:1441-6.

18 DeLong ER, DeLong DM, Clarke-Pearson DL. Comparing the areas under two or more correlated receiver operating characteristic curves: a nonparametric approach. Biometrics 1988;44:837-45.

19 Lemeshow S, Hosmer DW. A review of goodness of fit statistics for use in the development of logistic regression models. Am J Epidemiol 1982;115:92-106.

20 Redelmeier DA, Bloch DA, Hickam DH. Assessing predictive accuracy: how to compare Brier scores. J Clin Epidemiol 1991:44:1141-6.

21 Pencina MJ, D'Agostino RB, D'Agostino RB, et al. Evaluating the added predictive ability of a new marker: from area under the ROC curve to reclassification and beyond. Stat Med 2008;27:157-72.

22 Pugh RN, Murray-Lyon IM, Dawson JL, et al. Transection of the oesophagus for bleeding oesophageal varices. Br J Surg 1973;60:646-9.

23 Heuman DM, Mihas AA, Habib A, et al. MELD-XI: a rational approach to "sickest first" liver transplantation in cirrhotic patients requiring anticoagulant therapy. Liver Transp/ 2007;13:30-7.

24 Hiraoka A, Kumada T, Michitaka K, et al. Newly proposed ALBI grade and ALBI-T score as tools for assessment of hepatic function and prognosis in hepatocellular carcinoma patients. Liver Cancer 2019;8:312-25.

25 Kim MS, Kato TS, Farr M, et al. Hepatic dysfunction in ambulatory patients with heart failure: application of the MELD scoring system for outcome prediction. J Am Coll Cardiol 2013;61:2253-61.

26 Abe S, Yoshihisa A, Takiguchi M, et al. Liver dysfunction assessed by model for end-stage liver disease excluding Inr (MELD-XI) scoring system predicts adverse prognosis in heart failure. PLOS One 2014;9:e100618.

27 Inohara T, Kohsaka S, Shiraishi Y, et al. Prognostic impact of renal and hepatic dysfunction based on the MELD-XI score in patients with acute heart failure. Int J Cardiol 2014;176:571-3.

28 Biegus J, Zymliński R, Sokolski M, et al. Impaired hepato-renal function defined by the MELD XI score as prognosticator in acute heart failure. Eur J Heart Fail 2016;18:1518-21.

29 Grodin JL, Gallup D, Anstrom KJ, et al. Implications of alternative hepatorenal prognostic scoring systems in acute heart failure (from DOSE-AHF and ROSE-AHF). Am J Cardiol 2017;119:2003-9. 
30 Aimo A, Januzzi JL, Mueller C, et al. Admission high-sensitivity troponin $\mathrm{T}$ and NT-proBNP for outcome prediction in acute heart failure. Int J Cardiol 2019;293:137-42.

31 Scrutinio D, Ammirati E, Guida P, et al. Clinical utility of N-terminal pro-B-type natriuretic peptide for risk stratification of patients with acute decompensated heart failure. derivation and validation of the ADHF/NT-proBNP risk score. Int J Cardiol 2013;168:2120-6.

32 Zoair AM, Abdel-Hafez MA, Mawlana W, et al. Serum levels of Nterminal-pro B-type natriuretic peptide as a diagnostic marker for left ventricular dysfunction in children with end-stage renal disease on hemodialysis. Saudi J Kidney Dis Transpl 2016;27:1114-22.

33 Artunc F, Mueller C, Breidthardt T, et al. Comparison of the diagnostic performance of three natriuretic peptides in hemodialysis patients: which is the appropriate biomarker? Kidney Blood Press Res 2012;36:172-81.

34 Giallourakis CC, Rosenberg PM, Friedman LS. The liver in heart failure. Clin Liver Dis 2002;6:947-67.

35 Shao L, Han B, An S, et al. Albumin-to-bilirubin score for assessing the in-hospital death in cirrhosis. Trans/ Gastroenterol Hepatol 2017;2:88.

36 Henrion J, Schapira M, Luwaert R, et al. Hypoxic hepatitis: clinical and hemodynamic study in 142 consecutive cases. Medicine 2003:82:392-406.

37 Henrion J, Descamps O, Luwaert R, et al. Hypoxic hepatitis in patients with cardiac failure: incidence in a coronary care unit and measurement of hepatic blood flow. J Hepatol 1994;21:696-703.

38 Wu J-M, Skill NJ, Maluccio MA. Evidence of aberrant lipid metabolism in hepatitis $\mathrm{C}$ and hepatocellular carcinoma. HPB 2010;12:625-36.
39 Perez-Matos MC, Sandhu B, Bonder A, et al. Lipoprotein metabolism in liver diseases. Curr Opin Lipidol 2019;30:30-6.

40 Krautbauer S, Wiest R, Liebisch G, et al. Associations of systemic sphingolipids with measures of hepatic function in liver cirrhosis are related to cholesterol. Prostaglandins Other Lipid Mediat 2017;131:25-32.

41 Lei $\mathrm{Q}$, Zhang $\mathrm{Y}, \mathrm{Ke} \mathrm{C}$, et al. Value of the albumin-bilirubin score in the evaluation of hepatitis B virus-related acute-on-chronic liver failure, liver cirrhosis, and hepatocellular carcinoma. Exp Ther Med 2018;15:3074-9.

42 Xavier SA, Vilas-Boas R, Boal Carvalho P, et al. Assessment of prognostic performance of albumin-bilirubin, Child-Pugh, and model for end-stage liver disease scores in patients with liver cirrhosis complicated with acute upper gastrointestinal bleeding. Eur $J$ Gastroenterol Hepatol 2018;30:652-8.

43 Chen B, Lin S. Albumin-Bilirubin (ALBI) score at admission predicts possible outcomes in patients with acute-on-chronic liver failure. Medicine 2017;96:e7142.

44 Hiraoka A, Kumada T, Kudo M, et al. Albumin-Bilirubin (ALBI) grade as part of the evidence-based clinical practice guideline for HCC of the Japan Society of hepatology: a comparison with the liver damage and Child-Pugh classifications. Liver Cancer 2017;6:204-15.

45 Wang Y, Tuomilehto J, Jousilahti P, et al. Serum $\gamma$-glutamyltransferase and the risk of heart failure in men and women in Finland. Heart 2013;99:163-7.

46 Nikolaou M, Parissis J, Yilmaz MB, et al. Liver function abnormalities, clinical profile, and outcome in acute decompensated heart failure. Eur Heart J 2013;34:742-9. 